\title{
Gastrointestinal pharmacology: challenges ahead
}

\author{
Gareth J Sanger* \\ Wingate Institute of Neurogastroenterology, Barts and The London School of Medicine and Dentistry, Queen Mary University of London, London, UK \\ *Correspondence: g.sanger@qmul.ac.uk
}

Definitions are important and help shape how medical science develops. The Latin name 'materia medica' described therapeutically-useful materials (plants, animals and minerals) and the term evolved into the word 'pharmacology', defined by the British Pharmacology Society as the study of drugs and the way in which they work. In this new journal we need to broaden the definition to include the functions of naturally-occurring mediators such as neurotransmitters, cytokines and hormones, 'tool compounds' and the development of structure-activity relationships, the characterization of new antibodies and fusion proteins, and even the actions of bacteria, provided the cell-cell interactions can be characterized.

The gastrointestinal (GI) tract is an extraordinary world. It represents a huge interface between the external environment and the systems which sustain our existence (the surface area of mucosal villae of the human gut is equivalent to that of a football pitch). It helps orchestrate the delivery of food into itself and assists in how this is utilized. Thus, as the largest endocrine organ in the body, the gut uses hormones together with its own intrinsic and extrinsic nervous systems, to help promote appetite (eg. via the release of ghrelin and other hormones from the stomach) and potentiate glucose-dependent insulin secretion from islet $\beta$-cells (via GLP-1; glucagon-like peptide 1). It defends itself from bad selections of food, sometimes by releasing 5 -HT ( $>90 \%$ of total mammalian body 5 -HT is found in enterochromaffin cells where it can induce vomiting or diarrhea) and always via its own extensive immune system (the number of lymphocytes within the gutassociated lymphoid system for example, is roughly equivalent to the spleen). Digestion begins with an incredibly toxic mix of acid and proteases in the stomach. Movements of the GI tract are controlled mostly by the intrinsic (enteric) nervous system which in terms of numbers of nerve cells is comparable to the spinal cord. Control is further achieved by a large number of different GI hormones, operating on the enteric and vagal nerve systems to modulate GI movements as well as influence satiety and certain aspects of metabolism. Final stages of digestion and absorption take place in the colon where the huge colony of bacteria (the bacterial gene compliment of the gut exceeds that of the human body, with a metabolic capacity in the same range as the liver) ferment unabsorbed carbohydrates and proteins. The bacteria also have a symbiotic relationship with the mammalian body, affecting colonic development, inflammation and factors as diverse as weight gain and anxiety; potentially harmful interactions between the bacteria and the gut are regulated by the immune system but breakdowns in regulation can lead to disease.

The complex interactions between different systems of the gut and between the gut and the rest of the body suggest that it is not always reasonable to study these different mechanisms in isolation. One challenge to GI scientists, faced with organizational 'reductionism' is to remember this complexity and integrate disciplines such as 'neuroscience', 'immuno-inflammation' and 'metabolism' (and even 'bacteriology'), back into gastroenterology. Failure to do so slows down the recognition that some pathological conditions which are not obviously gastrointestinal in origin can, however, be controlled by modulating GI function. A simple and dramatic example of what has been missed, until recently, is the discovery that diabetes in the obese can be reduced by surgical rearrangement of the gut, so food is made to bypass most of the stomach and upper small intestine.

There is always a need to embrace new technologies and a second challenge for GI pharmacologists is to stay involved. To some extent, the GI functions of recently 'de-orphanised' G-protein-coupled receptors have been investigated, but much remains to be done; the same is also true for the wide range of innate and adaptive mucosal immune mechanisms which still need to be evaluated in the gut. GI pharmacologists need to be involved in the characterization of monoclonal antibodies or Fc-fusion proteins used to modify GI functions (especially when the immunoglobulin is fused to the binding region of the receptor). Further, as major advances in epigenetics are beginning to open new ways in which complex functional disorders (such as irritable bowel syndrome) might be treated, GI pharmacologists have a role to play in characterizing compounds which manipulate epigenetic modifications; assays need to be designed, for example, to demonstrate efficacy in native, therapeutically-relevant cell systems. Equally challenging is the need to find ways of defining the mechanisms by which probiotic formulations or specific bacteria affect GI function and then design assays to characterize the actions of one type of bacteria - or bacterial product over another.

This is a GI pharmacology journal. It can help 'bridge the gap' between gastroenterology and pharmacology, by describing the new science which is sometimes too specialized or too immature to find its way into gastroenterology journals, yet is relevant to GI science. It must also be inclusive and bring all relevant subspecialties of GI science onto a single gastrointestinal platform. It needs to cross traditional boundaries of 'neuroscience', 'immunology' and 'metabolism', so studies on gastric acid secretion, gastrointestinal motility and secretion sit alongside studies on mechanisms of GI sensations (such as taste, appetite, nausea and pain), immune regulation and inflammation. All aspects of translation will be important, including comparisons between recombinant and native receptor functions, between animal and human biology and between in vitro predictions and in vivo efficacy. The latter, for example, remains a critical 
issue, in view of the continued use of animal models of pain in spite of their failure to translate across to the clinic.

In conclusion, three over-riding challenges have been identified. There is a need to remember that the GI tract does not exist in isolation but has major influences on the rest of the body. There is a need to embrace new technologies, some of which promise to revolutionize how we study GI functions and disease. Finally, there is a need to build a platform in which GI pharmacologists can come together. It is to be hoped that this journal will play at least some role in meeting this challenge.

Received:08 April 2010; accepted:09 April 2010; published online: 21 May 2010.
Citation: Sanger GJ (2010) Gastrointestinal pharmacology: challenges ahead. Front. Pharmacol. 1:4. doi: 10.3389/ fphar.2010.00004

This article was submitted to Frontiers in Gastrointestinal Pharmacology, a specialty of Frontiers in Pharmacology. Copyright () 2010 Sanger. This is an open-access article subject to an exclusive license agreement between the authors and the Frontiers Research Foundation, which permits unrestricted use, distribution, and reproduction in any medium, provided the original authors and source are credited. 